\title{
Importance of Preoperative Knowledge of the Biomarker HE4 in Early-stage Endometrial Cancer Regarding Surgical Management
}

\author{
JIRI PRESL ${ }^{1}$, PETRA OVESNA ${ }^{2}$, ZDENEK NOVOTNY ${ }^{1}$, PAVEL VLASAK ${ }^{1}$, JIRI BOUDA $^{1}$, JAN KOSTUN ${ }^{1}$, \\ ONDREJ TOPOLCAN ${ }^{3}$, RADEK KUCERA $^{3}$, MARKETA BEDNARIKOVA $^{4}$ and VIT WEINBERGER ${ }^{5}$ \\ ${ }^{1}$ Department of Gynecology and Obstetrics, University Hospital Pilsen, Pilsen, Czech Republic; \\ ${ }^{2}$ Institute of Biostatistics and Analyses, Faculty of Medicine, Masaryk University, Brno, Czech Republic; \\ ${ }^{3}$ Laboratory of Immunoanalysis, Department of Nuclear medicine, \\ University Hospital in Pilsen, Pilsen, Czech Republic; \\ ${ }^{4}$ Clinic of Internal Medicine - Hematology and Oncology, \\ University Hospital in Brno and Masaryk University, Brno, Czech Republic; \\ ${ }^{5}$ Department of Gynecology and Obstetrics, \\ University Hospital in Brno and Masaryk University, Brno, Czech Republic
}

\begin{abstract}
Aim: To analyze the utility of HE4 assessment in preoperative management of patients with early-stage endometrial cancer for stratification into low-risk and highrisk groups. Patients and Methods: The following data were prospectively collected from patients operated for endometrial cancer from 05/2012 till 9/2016; age, HE4, CA125, expert ultrasound examination of the pelvis, histotype, grade, FIGO stage. Results: In total, 124 patients were enrolled. A cut-off of $\geq 113$ pmol/l HE4 demonstrated $40.3 \%$ sensitivity and $83.9 \%$ specificity for detection of high-risk patients. Correlations of HE4 with age $(p<0.001)$, depth of myometrial invasion $(p=0.001)$, clinical stage of the disease according to ultrasound - Tla vs. T1b (63.6 pmol/l vs. $110.6 \mathrm{pmol} / \mathrm{l}, \mathrm{p}<0.001)$ were found. However, no correlation of HE4 with lymph node invasion ( $p=0.07)$ and tumor grade $(p=0.212)$ was identified. Conclusion: HE4 levels correspond to clinical and FIGO stage of the disease. The sensitivity and specificity does not reach the transvaginal ultrasound results in preoperative assessment of the extent of the disease. Combination of HE4 with ultrasound does not improve the stratification of patients into low-risk and high-risk groups. Preoperative assessment of HE4 is useful providing no imaging method is available.
\end{abstract}

Correspondence to: Vit Weinberger, MD, Ph.D., Department of Gynecology and Obstetrics, University Hospital Brno and Masaryk University, Obilni trh 11, Brno, 60200, Czech Republic. Tel: +420 532238306, e-mail: vit.weinberger@gmail.com

Key Words: CA125, HE4, endometrial cancer, preoperative staging.
Endometrial cancer (EC) patients often exhibit signs and symptoms, such as postmenopausal bleeding that lead to diagnosis of the malignancy in early stages of the disease. As a result, more than $70 \%$ of EC cases are diagnosed at an early stage with a favorable prognosis (1). Abdominal ultrasound (US) combined with transvaginal ultrasound of the pelvis (TVUS) belong to obligatory imaging methods for EC staging in the Czech Republic. Histological type and grade of differentiation of the tumor are assessed from a biopsy obtained by dilatation and curettage of the uterus or hysteroscopy. The differentiation between carcinomas associated with good or poor prognosis helps selecting patients that would benefit from more aggressive primary treatment or adjuvant therapy.

In addition to established clinical parameters, biomarker(s) for better risk stratification in high-risk or low-risk EC could improve individualized primary treatment and would help avoid over- or undertreatment of EC patients. Studies investigating the prognostic value of CA125 in EC patients have revealed conflicting results. While Sood et al. (2) described preoperatively elevated CA125 levels as an independent prognostic marker for poor survival, other reports have shown no correlation between CA125 levels and extent of the disease (3-6). Moore et al. (7) showed the superiority of HE4 over CA125 in the detection of EC, especially in early stages. Recently, a study demonstrated a putative predictive role of HE4 in EC (8). Prognostic significance of serum HE4 has been demonstrated for patients with poorly differentiated endometrial cancer $(9,10)$. However, no serum biomarker is currently used in the management of EC owing to the lack of data and knowledge of relevant cut-offs (11). 
The purpose of this study was to evaluate the clinical significance of preoperative assessment of serum levels of HE4 in differentiation between low- and high-risk EC patients. The secondary objective of this study was to evaluate the correlation of HE4 with clinical (according to preoperative ultrasound) and pathologic extent of the disease (IA versus IB and higher stages, lymph node involvement) as well as with grading and histotype of the tumor. Another objective for clinical practice was answering the question, whether the preoperative knowledge of HE4 level will add value to expert ultrasound and improve patient stratification in low- or high-risk groups.

\section{Patients and Methods}

This was a single-Centre prospective study performed from April 2012 to September 2016. Patients with probable early stage EC according to previous staging examinations (US, chest X-ray, endometrial biopsy) were enrolled. Only patients with histologically-proven primary EC from uterine curettage or hysteroscopy preoperatively were included in the study. All patients underwent a pre-operative ultrasound staging examination according to the methodology and protocol of our institution (12) and blood samples for CA125 and HE4 were collected and examined. The extent of the surgery was based on the staging ultrasound examination and knowledge of histotype and grading. Type A radical hysterectomy with bilateral salpigoophorectomy was performed in all patients (13). Systematic pelvic and paraaortic lymphadenectomy was included where indicated.

Age, preoperative levels of CA125 and HE4, results of TVUS with respect to depth of myometrial invasion and cervical involvement, lymphadenopathy, and pathological data from biopsies (type of the tumor, grading) were recorded. Final pathological data (stage, histotype and grade) were assessed post-operatively according to the International Federation of Gynecology and Obstetrics 2009 staging system (FIGO) and the World Health Organization 2003 (14) pathologic classification of female reproductive organs. The patients were divided into three groups for statistical evaluation. Low risk group was defined as FIGO IA, grade 1-2 according to preoperative investigations. The systematic pelvic (PLN) and paraaortic lymphadenectomy (PALN) was not performed in these cases. Intermediate risk group was defined as FIGO stage IB, G1-2. In this case, the systematic PLN and PALN are not obligatory and were considered according to the perioperative circumstances. The systematic PLN and PALN were always performed in high risk group, i.e. FIGO IA and IB, G3 endometroid and non-endometroid histotype and all clinically more advanced stages. The differentiation between low and high risk patients is a key for clinical practice; therefore, ROC (Receiver Operating Characteristic) analysis was employed for the assessment of the optimal HE4 cut-off value. In the first analysis, the group of patients with intermediate-risk carcinoma was joined with the low-risk while, the second analysis was performed joining intermediate-risk carcinoma to high-risk group. Cut-off levels for CA125 and HE4 markers for both scenarios were determined; sensitivity and specificity for differentiation of a low- and high-risk group were calculated. This study was approved by the local ethics committee, and all patients gave their informed consent.

Preoperative ultrasound examination. Each patient underwent both transabdominal and transvaginal US scan within 14 days before admission in the operating theater by one of the two oncogynecologists experienced in the field of US diagnostics in gynecologic oncology. They were expert sonographers with gynecologic oncology experience and national certification. Each US examination was immediately described in the written report; these reports were used for the study analysis. Descriptions and examination reports were based on the standards applied by our center (15). During US staging examination of uterine cavity, myometrium, cervix and pelvic lymph nodes were carefully assessed in every patient to describe the extent of the tumor locally $(16,17)$.

Quantitative determination of HE4 and CA125 in human serum. Blood was collected prior to surgery and after diagnostic curettage. The sampling was always performed from the cubital vein using VACUETTE $^{\circledR}$ blood collection tubes (Greiner Bio-One, Austria) between 7-10 am. Serum was separated by centrifugation for 10 minutes at $1,700 \times g$ and the samples were analyzed either immediately or stored frozen at $-80^{\circ} \mathrm{C}$ until the analysis. Levels of both markers were determined using a chemiluminescence method by Architect 1000i (Abbott, USA) in Immunoanalysis laboratory of the University Hospital Pilsen.

Statistical analysis. Original values of HE4 and CA125 markers were transformed with log-function due to their log-normal distribution. Geometric mean and $95 \%$ confidence interval (CI) after back transformation are presented in the results. All other parameters had normal distribution of values. Student $t$-test or ANOVA with Tukey post-hoc test were employed for the comparison of HE4 in different two or more groups (clinical parameters). Relation between HE4 and age was assessed using Pearson correlation. ROC (Receiver Operating Characteristic) analysis was applied to determine the best cut-off to distinguish patients into low-risk and high-risk group. A cut-off with the highest sum of sensitivity and specificity was chosen. All analyses were performed using SPSS Statistics 23, all tests were done as two-sided at a level of significance of 0.05 .

\section{Results}

In total, 124 patients with EC were enrolled in the study, all operated in the Center for Gynecologic Oncology of the University Hospital in Pilsen. The FIGO stage of the disease was determined from the final histology report postoperatively. In the studied group, 70 patients were in FIGO stage IA, 44 FIGO stage IB, and none FIGO stage II. A total of 10 patients had retroperitoneal lymph nodes involvement; pelvic lymph nodes in six cases - FIGO IIIC1; paraaortic lymph nodes in four patients - FIGO IIIC2. Pathomorphologic and sonographic characteristics of the groups are provided in Table I. Median preoperative HE4 and CA125 levels in the studied group of patients with early stage endometrial cancer were 75.5 and 18.0 respectively. The patients with very low HE4 level had a lowrisk carcinoma; conversely patients with extremely high HE4 level had an intermediate or high-risk carcinoma (Table II). Paradoxically, the highest HE4 levels were observed in intermediate-risk group of patients. No clear HE4 cut-off for differentiation of risk groups could be determined. The most patients were diagnosed at the stage of disease IA or IB. 
Table I. Pathomorphologic and sonographic characteristics of the groups.

\begin{tabular}{lc}
\hline & $\mathrm{N}(\%)$ \\
\hline Age (years), mean (SD) & $67.6(9.8)$ \\
Clinical staging by TVUS & \\
T1aNxM0 & $80(64.5 \%)$ \\
T1bNxM0 & $39(31.5 \%)$ \\
T2NxM0 & $2(1.6 \%)$ \\
T3aNxM0 & $2(1.6 \%)$ \\
T0 & $1(0.8 \%)$ \\
FIGO & \\
IA & $70(56.5 \%)$ \\
IB & $44(35.5 \%)$ \\
IIIC1 & $6(4.8 \%)$ \\
IIIC2 & $4(3.2 \%)$ \\
Depth of tumor invasion into the myometrium & \\
$<50 \%$ & $71(57.3 \%)$ \\
$>50 \%$ & $53(42.7 \%)$ \\
Histologic type & \\
Endometroid adenoca & $110(88.7 \%)$ \\
Clear cell adenoca & $5(4 \%)$ \\
Mixed (adeno, squamous) & $1(0.8 \%)$ \\
High-grade serous adenoca & $6(4.8 \%)$ \\
Mucinous adenoca & $1(0.8 \%)$ \\
Grade & $65(52.4 \%)$ \\
1 & $32(25.8 \%)$ \\
2 & $27(21.8 \%)$ \\
3 &
\end{tabular}

In the first analysis, (intermediate-risk patients were added to low-risk group) only $35.5 \%$ of patients with high-risk carcinoma with a specificity of $75.3 \%$ were identified with cut-off $\mathrm{HE} 4 \geq 115$. Regarding CA125, the best cut-off calculated by ROC analysis was CA125 $\geq 30$, providing sensitivity $48.4 \%$ and specificity $80.6 \%$. In second scenario, (intermediate-risk patients were added to high-risk group) cut-off $\mathrm{HE} 4 \geq 113$ was identified with the sensitivity of $40.3 \%$ and specificity of $83.9 \%$ to determinate these two risk groups (Figure 1). Cut-off CA125 $\geq 15$ demonstrated $75.8 \%$ sensitivity and $53.2 \%$ specificity.

We focused on the correlation of serum HE4 level with patient age, preoperative TVUS, tumor histotype, grading and lymph node involvement. HE4 correlated well with patient age $(p<0.001)$ (Figure 2) and preoperative TVUS, where statistically significant differences between patients stage $\mathrm{T} 1 \mathrm{a}$ and $\mathrm{T} 1 \mathrm{~b}$ were observed. The mean values for HE4 for clinical stage T1a reached $63.6 \mathrm{pmol} / 1$ (95\% CI, 21.6-187.6) vs. $110.6 \mathrm{pmol} / \mathrm{l}(95 \%$ CI, 34.8-350.9) for T1b, $p<0.001$ (Table III). Furthermore, a correlation between HE4 and histological depth of myometrial invasion was found; the HE4 levels for stage FIGO IA vs. IB were $63.2 \mathrm{pmol} / 1$ (95\% CI=21.9-182.1) vs. $99.7 \mathrm{pmol} / 1$ (95\% $\mathrm{CI}=27.3-363.9)$ respectively, $p=0.001$. Correlation with FIGO staging FIGO IA and IIIC2 was also found $63.2 \mathrm{pmol} / 1$ (95\% $\mathrm{CI}=21.9-182.1)$ and $164.9 \mathrm{pmol} / \mathrm{l} \quad(95 \% \quad \mathrm{CI}=35.8-759.6)$
Table II. HE4 (pmol/l) according to the risk group of carcinoma.

\begin{tabular}{lccc}
\hline & $\mathrm{N}$ & Mean & $95 \% \mathrm{CI}$ \\
\hline Low risk & 62 & 64.7 & $21.5-194.7$ \\
Intermediate & 31 & 100.9 & $25.8-394$ \\
High risk & 31 & 86.3 & $25.9-287.7$ \\
Total & 124 & 77.7 & $22.4-269.3$ \\
\hline
\end{tabular}

Table III. HE4 (pmol/l) and clinical staging by TVUS and FIGO.

\begin{tabular}{lccc}
\hline & $\mathrm{N}$ & Mean & $95 \% \mathrm{CI}$ \\
\hline Clinical staging by TVUS & & & \\
T1aNxM0 & 80 & 63.6 & $21.6-187.6$ \\
T1bNxM0 & 39 & 110.6 & $34.8-350.9$ \\
T2NxM0 & 2 & 112.1 & $18.9-664.2$ \\
T3aNxM0 & 2 & 211.0 & $29.7-$ \\
1498.9 & & & \\
T0 & 1 & 47.0 & - \\
FIGO & & & \\
IA & 70 & 63.2 & $21.9-182.1$ \\
IB & 44 & 99.7 & $27.3-363.9$ \\
IIIC1 & 6 & 84.2 & $36.7-193.2$ \\
IIIC2 & 4 & 164.9 & $35.8-759.6$ \\
\hline
\end{tabular}

respectively, $p=0.01$ (Table III). The HE4 level did not correlate with tumor grade, following mean values were observed; G1 70.7, G2 87.8 and G3 $84.5 \mathrm{pmol} / 1, p=0.212$. The correlation of HE4 with lymph nodes involvement did not reach statistical significance $(p=0.07)$ in spite of the fact that patients with positive lymph nodes had higher HE4 levels (mean=110.2) than those with no lymph node involvement (mean=75.4 pmol/l).

\section{Discussion}

There is a need for EC markers that can help stratify patients preoperatively into high-and low-risk categories to assist gynecologic oncology surgeon in selection of patients that can benefit from lymphadenectomy, hysterectomy or radical hysterectomy, or extensive surgery. A number of recent studies demonstrate that the serum biomarker HE4 is increased not only in ovarian cancer, but also in EC, type I and II $(9,18)$. Studies on patients with EC have shown that serum HE4 levels correlated with myometrial invasion, grading, advanced stage disease and with the presence of extrauterine disease, such as lymphatic and peritoneal metastases (19). Other studies found no or only a partial correlation $(8,9,20)$. No clear cut-off value for HE4 serum levels has been defined for EC patients (21, 22). In the study of Mutz-Dehbalaie et al. from 2012, investigators established the cut-off level for discrimination between high and low HE4 serum levels based on median value $(81 \mathrm{pmol} / \mathrm{l})$. Serum HE4 median values in EC patients 


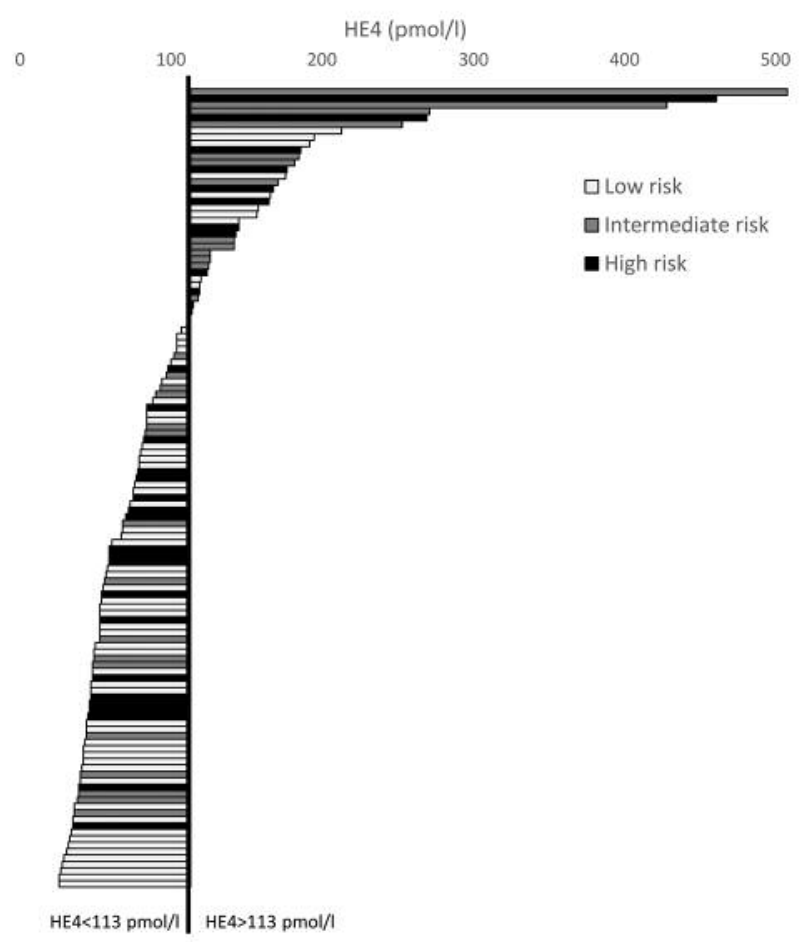

Figure 1. Classification of patients according to HE4 cut-off 113 pmol/l. Each patient is represented by one horizontal bar. The best cut-off for HE4 113 pmol/l devides all values into two group. Patients with HE4 $<113 \mathrm{pmol} / \mathrm{l}$ are on the left side; patients with $\mathrm{HE} 4 \geq 113 \mathrm{pmol} / \mathrm{l}$ are on the right side. White bars on the left sides depict correctly predicted patients, i.e. low risk, whereas grey and black bars (intermediate or high risk, respectively) represent wrongly predicted patients (false negative). White bars on the right side represent wrongly predicted patients (false positive). It is evident that patients with intermediate risk (grey bars) have higher values of HE4, thus these patients were joined to high risk group.

were similar in our study (median $75.5 \mathrm{pmol} / \mathrm{l}$ ) as compared to previous published investigations (7-9). Median $75.5 \mathrm{pmol} / 1$ was affected by the selection bias as primarily advanced stages of disease were not included in the study. The distribution of median CA125 values corresponded to the low, intermediate and high risk categories. On the contrary, in case of HE4, the intermediate risk group paradoxically showed higher values than high-risk group of patients (Table II). The importance of this finding is unclear. It may be due to the random error with low number of patients. On the other hand, it may point to a group of patients with a different prognostic significance, i.e. different disease-free interval or overall survival.

The HE4 cut-off for risk group differentiation was calculated for the situations when the intermediate risk group was added to the low-risk group or the high-risk group. At the cut-off HE4 $\geq 113$ we identified $40.3 \%$ patients in high/intermediate risk. If this cut-off was used routinely in preoperative evaluation, then $16.1 \%$ patients would be operated radically in spite of having a low-risk carcinoma. The correlation between high HE4 levels

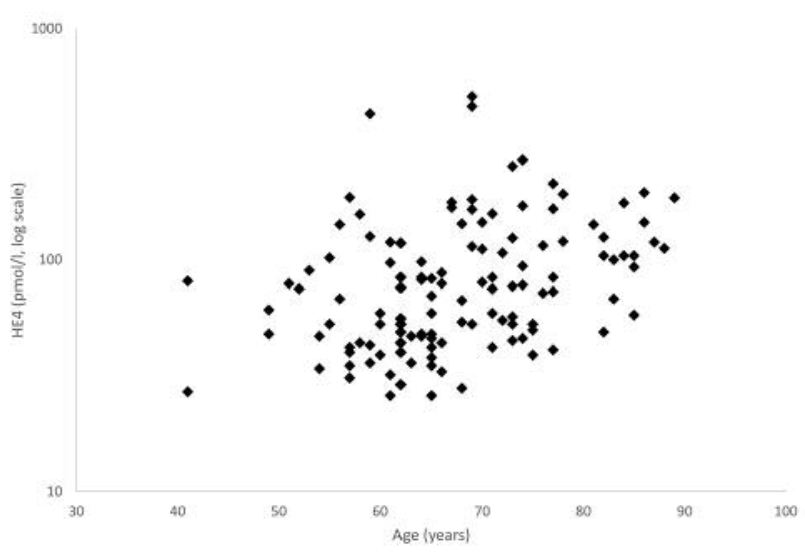

Figure 2. Correlation between HE4 and age. Relation between age and HE4 is evident - older patients higher HE4.

and myometrial invasion was reported previously $(8,20)$. Both studies described significantly elevated HE4 serum levels in patients with more than $50 \%$ myometrial invasion compared to patients with less than 50\% myometrial invasion. Our results are in agreement with these reports regarding the association of HE4 and depth of myometrial invasion (IA vs. IB and higher, $p=0.001$ ) (Table III). Given the fact that deep myometrial invasion increases the likelihood of lymph node metastasis, HE4 levels could provide an additional preoperative information concerning the benefit of lymphadenectomy. Bignotti et al. described that higher HE4 levels were significantly associated with positive lymph nodes and presence of lymphovascular invasion (9). Additionally, a recent study revealed that HE4 expression is associated with lymph node metastases in breast cancer (23). Interestingly, neither Moore et al. (8) nor our study $(p=0.07)$ found an association between HE4 serum levels and absence or presence of lymph node involvement. The result can be biased by the low number of patients with positive lymph nodes in our study $(n=10)$. The correlation of HE4 with histologic type of the tumor could not be calculated due to major representation of endometroid carcinoma in our study group, which copies the clinical practice.

TVUS is associated with high sensitivity and specificity in identification of high-risk patients (78\% (95\% CI=63.7-88.0\%) and $89 \%$ (95\% CI=81.7-93.8\%) respectively) (24). It is capable of diagnosis of tumor invasion into the myometrium by the more than $50 \%$ of its thickness, the cervix, the uterine appendages or surrounding tissues with high sensitivity and specificity (25-27). Depth of myometrial invasion as a basic parameter of FIGO stage I, may be undervalued especially in small well differentiated tumors with low perfusion and conversely overvalued in poorly differentiated or undifferentiated tumors of larger dimensions with high perfusion (16). One of the objectives of our study was to assess the additional benefit of serum marker 


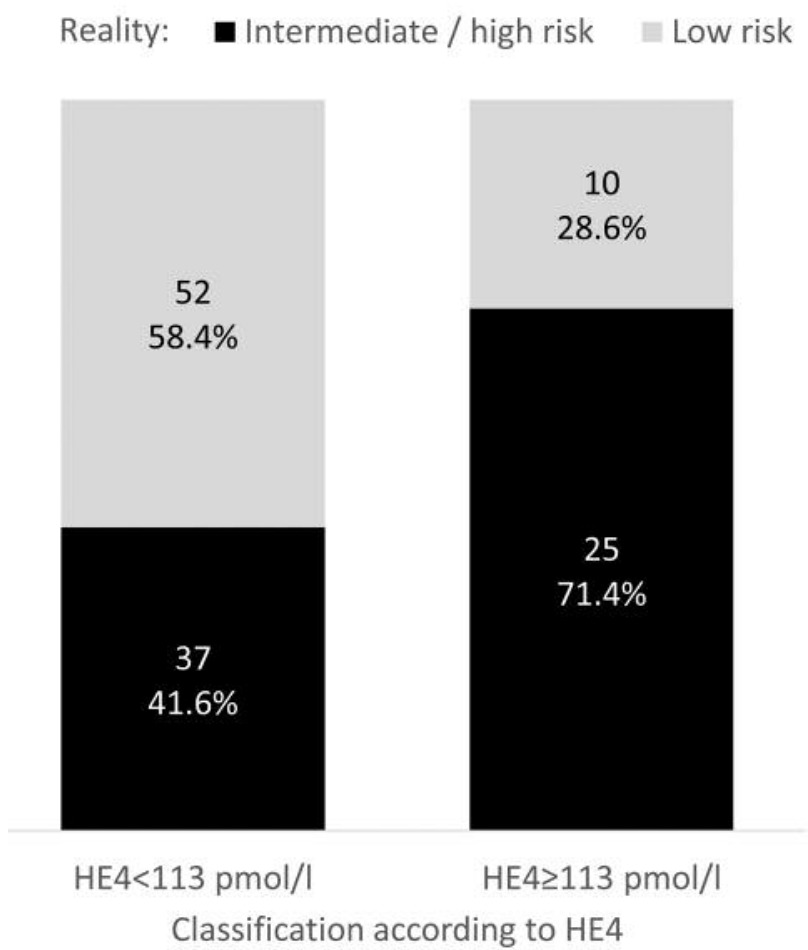

Figure 3. Classification of patients according to HE4 cut-off 113 pmol/l. Forty-seven (37.9\%) patients were classified wrongly according to HE4 - 37 of them were misclassified into low-risk group (HE<113 pmol/l) although they had intermediate or high-risk in reality (false negative); 10 of them were misclassified into high risk group (HE $\geq 113$ pmolll) although they had low risk in reality (false positive). The rest of 77 (62.1\%) patients were classified correctly.

HE4 use to pelvic TVUS in preoperative management of EC. At the cut-off HE4 $\geq 113,47$ (37.9\%) patients were misclassified 10 patients with low-risk carcinoma as high-risk patients and conversely 37 patients classified as low-risk had a high/intermediate cancer (Figures 1 and 3). On the contrary, TVUS let to misclassification of $32(25,8 \%)$ patients -25 patients with high/intermediate-risk carcinoma were clinically determined as having stage T1a and 7 patients were classified as T1b and had a low-risk carcinoma (Figure 4).

The current study has several limitations. Blood was collected after the initial curettage or hysteroscopy. The impact of curettage/hysteroscopy on HE4 serum levels is unknown to date, however, but serum HE4 median values were comparable to previously published results (7-9). All data come from one Center for Gynecologic Oncology and may be affected by random error due to low number of participants in some groups (number of patients in stage FIGO II, IIIA and IIIB). On the other hand the data were collected prospectively. The study design copies clinical practice; a patient with EC is stratified into the proper risk group using preoperative staging results and treated accordingly. According to our knowledge, no study

\section{Reality: - Intermediate / high risk Low risk}

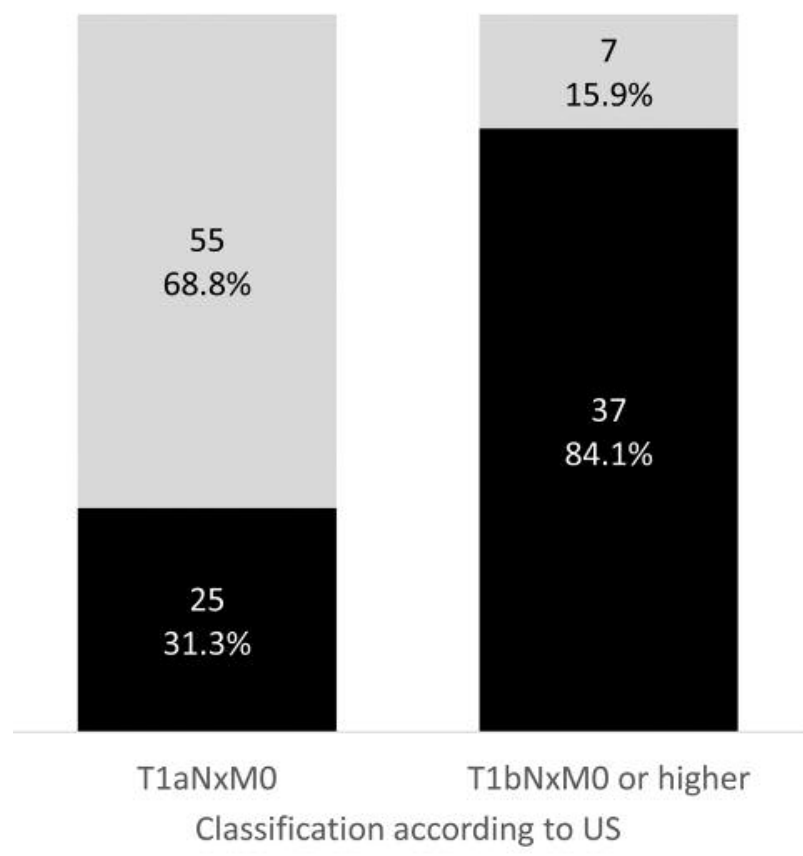

Figure 4. Classification of patients according to US. Thirty-two (25.8\%) patients were classified wrongly according to US - 25 of them were misclassified into low risk group by US (T1a) although they had intermediate or high risk in reality (false negative); 7 of them were misclassified into high-risk group (T1b or higher) although they had low risk in reality (false positive). The rest of 92 (74.2\%) patients were classified correctly.

has compared the reliability of the serum marker HE4 and preoperative TVUS.

\section{Conclusion}

The results of the study confirm a good correlation of HE4 with clinical staging using ultrasound and final FIGO staging by histopathology. HE4 correlates with depth of myometrial invasion, lymph node involvement and patient age. The determination of HE4 does not reach the sensitivity and specificity of an ultrasound examination and would not contribute to improvement in the stratification of a patient to proper risk group. According to our results, preoperative assessment of HE4 seems to be justifiable where no adequate imaging method for preoperative staging of the disease is available.

\section{Acknowledgements}

This work was supported by the Ministry of Health of the Czech Republic under Projects Fakultní nemocnice Plzeň - FNP1, 00669806) and CZ-DRO FNBr 65269705. 


\section{References}

1 Jemal A, Siegel R, Ward E, Murray T, Xu J, Smigal C and Thun MJ: Cancer statistics, 2006. CA Cancer J Clin 56: 106-130, 2006.

2 Sood AK, Buller RE, Burger RA, Dawson JD, Sorosky JI and Berman M: Value of preoperative CA 125 level in the management of uterine cancer and prediction of clinical outcome. Obstet Gynecol 90: 441-447, 1997.

3 Lo SS, Cheng DK, Ng TY, Wong LC and Ngan HY: Prognostic significance of tumour markers in endometrial cancer. Tumour Biol 18: 241-249, 1997.

4 Price FV, Chambers SK, Carcangiu ML, Kohorn EI, Schwartz PE and Chambers JT: CA 125 may not reflect disease status in patients with uterine serous carcinoma. Cancer 82: 1720-1725, 1998.

5 Soper JT, Berchuck A, Olt GJ, Soisson AP, Clarke-Pearson DL and Bast RC: Preoperative evaluation of serum CA 125, TAG 72, and CA 15-3 in patients with endometrial carcinoma. Am J Obstet Gynecol 163: 1204-1209, 1990.

6 Chen Y, Ren Y-L, Li N, Yi X-F and Wang H-Y: Serum human epididymis protein $4 \mathrm{vs}$. carbohydrate antigen 125 and their combination for endometrial cancer diagnosis: a meta-analysis. Eur Rev Med Pharmacol Sci 20: 1974-1985, 2016.

7 Moore RG, Brown AK, Miller MC, Badgwell D, Lu Z, Allard WJ, Granai CO, Bast RC and Lu K: Utility of a novel serum tumor biomarker HE4 in patients with endometrioid adenocarcinoma of the uterus. Gynecol Oncol 110: 196-201, 2008.

8 Moore RG, Miller CM, Brown AK, Robison K, Steinhoff M and Lambert-Messerlian G: Utility of tumor marker HE4 to predict depth of myometrial invasion in endometrioid adenocarcinoma of the uterus. Int J Gynecol Cancer 21: 1185-1190, 2011.

9 Bignotti E, Ragnoli M, Zanotti L, Calza S, Falchetti M, Lonardi S, Bergamelli S, Bandiera E, Tassi RA, Romani C, Todeschini P, Odicino FE, Facchetti F, Pecorelli S and Ravaggi A: Diagnostic and prognostic impact of serum HE4 detection in endometrial carcinoma patients. Br J Cancer 104: 1418-1425, 2011.

10 Capriglione S, Plotti F, Miranda A, Lopez S, Scaletta G, Moncelli M, Luvero D, De Cicco Nardone C, Terranova C, Montera R and Angioli R: Further insight into prognostic factors in endometrial cancer: the new serum biomarker HE4. Expert Rev Anticancer Ther 17: 9-18, 2017.

11 Colombo N, Creutzberg C, Amant F, Bosse T, González-Martín A, Ledermann J, Marth C, Nout R, Querleu D, Mirza MR, Sessa $\mathrm{C}$ and ESMO-ESGO-ESTRO Endometrial Consensus Conference Working Group: ESMO-ESGO-ESTRO Consensus Conference on Endometrial Cancer: diagnosis, treatment and follow-up. Ann Oncol 27: 16-41, 2016.

12 Frühauf F, Dvořák M, Haaková L, Hašlík L, Herboltová $\mathrm{P}$, Chaloupková B, Kožnarová J, Kubešová B, Lukáčová I, Marek R, Neumannová H, Peschout R, Přibyl V, Sedláková I, Smažinka M, Svobodová P, Vančo M, Vlasák P, Weinberger V, Zikán M and Fischerová D: Ultrasound staging of endometrial cancer recommended methodology of examination. Ceska Gynekol 79: 466-476, 2014.

13 Querleu D and Morrow CP: Classification of radical hysterectomy. Lancet Oncol 9: 297-303, 2008.

14 FIGO Committee on Gynecologic Oncology: FIGO staging for carcinoma of the vulva, cervix, and corpus uteri. Int J Gynaecol Obstet 125: 97-98, 2014.
15 Fischerova D: Ultrasound scanning of the pelvis and abdomen for staging of gynecological tumors: a review. Ultrasound Obstet Gynecol 38: 246-266, 2011.

16 Fischerova D, Frühauf F, Zikan M, Pinkavova I, Kocián R, Dundr P, Nemejcova K, Dusek L and Cibula D: Factors affecting sonographic preoperative local staging of endometrial cancer. Ultrasound Obstet Gynecol 43: 575-585, 2014.

17 Eriksson LSE, Lindqvist PG, Flöter Rådestad A, Dueholm M, Fischerova D, Franchi D, Jokubkiene L, Leone FP, Savelli L, Sladkevicius P, Testa AC, Van den Bosch T, Ameye L and Epstein E: Transvaginal ultrasound assessment of myometrial and cervical stromal invasion in women with endometrial cancer: interobserver reproducibility among ultrasound experts and gynecologists. Ultrasound Obstet Gynecol 45: 476-482, 2015.

18 Galgano MT, Hampton GM and Frierson HF: Comprehensive analysis of HE4 expression in normal and malignant human tissues. Mod Pathol 19: 847-853, 2006.

19 Li J, Dowdy S, Tipton T, Podratz K, Lu W-G, Xie X and Jiang S-W: HE4 as a biomarker for ovarian and endometrial cancer management. Expert Rev Mol Diagn 9: 555-566, 2009.

20 Kalogera E, Scholler N, Powless C, Weaver A, Drapkin R, Li J, Jiang S-W, Podratz K, Urban N and Dowdy SC: Correlation of serum HE4 with tumor size and myometrial invasion in endometrial cancer. Gynecol Oncol 124: 270-275, 2012.

21 Abdalla N, Piórkowski R, Stanirowski P, Słomka A, Cendrowski $\mathrm{K}$ and Sawicki W: Assessment of levels of the tumor markers HE4 and CA125 considering staging, grading and histological types of endometrial cancer. Prz Menopauzalny 15: 133-137, 2016.

22 Capriglione S, Plotti F, Miranda A, Ricciardi R, Scaletta G, Aloisi A, Guzzo F, Montera R and Angioli R: Utility of tumor marker HE4 as prognostic factor in endometrial cancer: a single-center controlled study. Tumour Biol 36: 4151-4156, 2015.

23 Kamei M, Yamashita S-I, Tokuishi K, Hashioto T, Moroga T, Suehiro S, Ono K, Miyawaki M, Takeno S, Yamamoto S and Kawahara K: HE4 expression can be associated with lymph node metastases and disease-free survival in breast cancer. Anticancer Res 30: 4779-4783, 2010.

24 Alcázar JL, Pineda L, Caparrós M, Utrilla-Layna J, Juez L, Mínguez JA and Jurado M: Transvaginal/transrectal ultrasound for preoperative identification of high-risk cases in well- or moderately differentiated endometrioid carcinoma. Ultrasound Obstet Gynecol 47: 374-379, 2016.

25 Sawicki W, Spiewankiewicz B, Stelmachów J and Cendrowski $\mathrm{K}$ : The value of ultrasonography in preoperative assessment of selected prognostic factors in endometrial cancer. Eur J Gynaecol Oncol 24: 293-298, 2003.

26 Akbayir O, Corbacioglu A, Numanoglu C, Guleroglu FY, Ulker V, Akyol A, Guraslan B and Odabasi E: Preoperative assessment of myometrial and cervical invasion in endometrial carcinoma by transvaginal ultrasound. Gynecol Oncol 122: 600-603, 2011.

27 Savelli L, Ceccarini M, Ludovisi M, Fruscella E, De Iaco PA, Salizzoni E, Mabrouk M, Manfredi R, Testa AC and Ferrandina G: Preoperative local staging of endometrial cancer: transvaginal sonography $v s$. magnetic resonance imaging. Ultrasound Obstet Gynecol 31: 560-566, 2008. 